\title{
Refrigerantes Convencionais Versus Refrigerantes Orgânicos: Análise do Potencial Erosivo
}

\section{Conventional Soft Drinks Versus Organic Soft Drinks: Analysis of Erosive Potential}

\author{
João Paulo Gomes Martins ${ }^{1}$ \\ Ingrid Andrade Meira ${ }^{2}$ \\ Juliellen Luiz da Cunha ${ }^{3}$ \\ Andressa Feitosa Bezerra de Oliveira ${ }^{4}$ \\ Ana Maria Barros Chaves Pereira ${ }^{5}$
}

\section{RESUMO}

Objetivo: Avaliar e comparar o potencial erosivo de refrigerantes orgânicos e convencionais, disponíveis no mercado, utilizando as variáveis de $\mathrm{pH}$, titulação ácida (TA) e capacidade tampão (CT). Metodologia: Foram analisadas 14 bebidas industrializadas adquiridas nos supermercados da Cidade de João Pessoa/ $\mathrm{PB}$, sendo 7 orgânicas e 7 convencionais do mesmo sabor. As medições de $\mathrm{pH}$ e TA para o pH 5,5 e 7,0 foram realizadas em triplicata, em $50 \mathrm{ml}$ de cada bebida, imediatamente após a sua abertura. A CT foi calculada baseada nos valores de $\mathrm{pH}$ e titulação ácida para o $\mathrm{pH} 7,0$. Os dados foram analisados pelo teste ANOVA, seguido de Bonferroni e a correlação de Pearson, com significância de 5\%. Resultados: Diferença estatisticamente significante foi encontrada entre os grupos, para todas as variáveis analisadas $(\mathrm{p}<0,05)$. $\mathrm{O} \mathrm{pH}$ dos refrigerantes variou de 2,37 (água tônica convencional) a 3,35 (laranja convencional). Os refrigerantes orgânicos apresentaram valores maiores de

TA 7.0 do que os convencionais, exceto para a água tônica. Os maiores valores de TA e CT foram encontrados no sabor limão orgânico, enquanto, os menores registraram-se no de guaraná zero convencional. Conclusão: Todas as bebidas analisadas demonstraram potencial erosivo significativo pela análise das variáveis químicas. O refrigerante orgânico de limão apresentou-se como o mais erosivo devido baixo $\mathrm{pH}$, sua maior TA e CT, evidenciando que a saliva terá maior dificuldade de tamponá-lo no meio bucal.

\section{DESCRITORES}

Erosão Dentária. Refrigerantes. Esmalte Dentário.

\begin{abstract}
Objective: To evaluate and compare the erosive potential of commercially available organic and conventional soft drinks, using the variables $\mathrm{pH}$, acid titration (TA) and buffering capacity (CT). Methodology: Fourteen industrialized beverages purchased in the supermarkets of João Pessoa / PB were analyzed, 7 organic and 7 conventional with the same flavor. The $\mathrm{pH}$ measurements and TA for $\mathrm{pH} 5.5$ and 7.0 were performed in triplicate in $50 \mathrm{ml}$ of each drink, immediately after opening. The CT was calculated based on $\mathrm{pH}$ and acid titration values for $\mathrm{pH}$ 7.0. Data were analyzed by ANOVA, followed by Bonferroni, and Pearson correlation, with a significance of $5 \%$. Results: Statistically significant difference was found between groups for all variables analyzed $(p<0.05)$. The $\mathrm{pH}$ of soft drinks ranged from 2.37 (conventional tonic water) to 3.35 (conventional orange). Organic soft drinks had higher TA 7.0 values than conventional ones, except for tonic water. The highest TA and $C T$ values were found in organic lemon flavor, while the lowest TA and CT values were for conventional diet "guaraná". Conclusion: All beverages analyzed showed significant erosive power by analysis of chemical variables. The lemon organic soft drink presented itself as the most erosive due to its low $\mathrm{pH}$, its higher TA and CT, showing that saliva will have greater difficulty buffering it in the mouth.
\end{abstract}

\section{DESCRIPTORS}

Tooth Erosion. Soft Drinks. Dental Enamel.

${ }^{1}$ Bolsista PIBIC do curso de Odontologia, Centro de Ciências da Saúde, Universidade Federal da Paraíba - UFPB, João Pessoa, Paraíba, Brasil.

${ }^{2}$ Doutoranda no Programa de Pós-graduação em Odontologia, Departamento de Prótese e Periodontia, Faculdade de Odontologia de Piracicaba, Universidade de Campinas - Unicamp, Piracicaba - São Paulo, Brasil.

${ }^{3}$ Bolsista PIBIC do curso de Odontologia, Centro de Ciências da Saúde, Universidade Federal da Paraíba - UFPB, João Pessoa, Paraíba, Brasil.

${ }^{4}$ Professora Histologia, Titular do Departamento de Morfologia - CCS, Doutora em Odontologia, Universidade Federal da Paraíba - UFPB, João Pessoa, Paraíba, Brasil.

${ }^{5}$ Professora Histologia, Associado do Departamento de Morfologia - CCS, Doutora em Odontologia, Universidade Federal da Paraíba - UFPB, João Pessoa, Paraíba, Brasil. 
A erosão dentária é uma condição multifatorial que tem despertado o interesse da comunidade científica nos últimos anos ${ }^{1}$. Os dados de sua prevalência a nível mundial variam entre $12 \%$ e $62 \%$ nos diversos estudos ${ }^{2,3}$, dependendo da faixa etária. No Brasil, a erosão dentária encontra-se entre $15 \%$ a $53 \%^{3,4}$. Entretanto, não está claro se a prevalência tem realmente aumentado, ou se apenas o aumento no entendimento sobre erosão dentária tem auxiliado no diagnóstico precoce ${ }^{5}$. É evidente, no entanto, um significativo aumento das lesões de erosão nos estudos com crianças e adolescentes ${ }^{3,6,7}$. O estágio inicial da lesão erosiva é caracterizado pelo amolecimento do esmalte, que ocorre como resultado da desmineralização parcial da superfície dentária ${ }^{8}$. Clinicamente, observa-se uma perda de brilho na superfície do esmalte, com uma aparência opaca, podendo resultar em pequenas concavidades com exposição da dentina e até mesmo da polpa, quando em estágios mais avançados da doença ${ }^{9}$.

Etiologicamente, a lesão de erosão pode se desenvolver a partir da interação de fatores químicos, biológicos e comportamentais $^{5}$, que levam a uma desmineralização do tecido dental, por ação de ácidos de origem intrínseca ou extrínseca, sem o envolvimento de bactérias ${ }^{10,11}$. Os principais fatores etiológicos relacionados com a erosão são os extrínsecos que incluem: (a) dieta rica em alimentos e bebidas ácidas; (b) ingestão frequente de medicamentos com caráter ácido e/ou (c) exposição à ácidos presentes no meio ambiente ${ }^{11,12}$. Além destes, os fatores intrínsecos também se apresentam relevantes por estarem ligados à anorexia nervosa e bulimia, bem como a qualquer distúrbio gastroesofágico persistente que leva a regurgitação constante do suco gástrico no meio bucal ${ }^{9}$.

Como um dos principais fatores de desenvolvimento da erosão está na dieta, o estudo do potencial erosivo de bebidas, relacionadas com as medições de $\mathrm{pH}$, de titulação ácida, de capacidade tampão ${ }^{12}$, bem como, das concentrações de cálcio, fósforo e fluoreto ${ }^{13}$ são dados importantes a serem estudados, especialmente com o surgimento de novos produtos no mercado. Além destes, a temperatura, a adesão da bebida a estrutura dentária e o tempo de contato com o tecido dental também são determinantes importantes no processo de desenvolvimento da erosão causada por bebidas ácidas ${ }^{12}$.

Assim, vários estudos, clínicos e laboratoriais, foram desenvolvidos com o intuito de se determinar o potencial erosivo de diversas bebidas ácidas ${ }^{2,14,15}$. Entretanto, na literatura, não foram encontradas pesquisas que abordassem a análise do potencial erosivo dos refrigerantes orgânicos, uma parcela do mercado que tem ganhado representatividade no panorama atual. Atualmente, a conscientização tem levado ao consumo de produtos mais saudáveis com menor teor de gordura, sal, açúcar e conservantes, tais como os alimentos light e diet. A preocupação com o meio ambiente e o consumo sustentável tem dado espaço e abertura para o consumo de alimentos naturais e orgânicos ${ }^{11,16}$.

O termo orgânico é uma rotulagem indicativa de produtos que atendem às normas estabelecidas de produção orgânica e que estão certificados por uma estrutura ou autoridade de certificação devidamente constituída ${ }^{17}$. De acordo com o Ministério de 
Agricultura e Pecuária, a legislação brasileira considera um produto orgânico, seja ele in natura ou processado, aquele que foi obtido em sistema de processo extrativista sustentável e não prejudicial ao ecossistema local ${ }^{18}$. Assim, devido ao substancial aumento do interesse do consumidor pelos alimentos orgânicos, existe a necessidade de se conhecer o alcance das bases científicas para suas alegações de superioridade ${ }^{17}$.

Sendo assim, este estudo teve por objetivo avaliar e comparar o potencial erosivo de refrigerantes convencionais (não orgânicos) e orgânicos disponíveis no mercado da cidade de João Pessoa - PB, utilizando as variáveis $\mathrm{pH}$, titulação ácida e capacidade tampão. $\mathrm{A}$ hipótese deste estudo é a de que os refrigerantes orgânicos apresentam um menor potencial erosivo do que os refrigerantes convencionais.

\section{METODOLOGIA}

Seleção das bebidas

Para esse estudo, foram escolhidos 14 tipos de refrigerantes industrializados, de diferentes marcas e sabores, sendo 7 deles refrigerantes orgânicos e os outros 7 , refrigerantes convencionais ou não orgânicos, disponíveis no mercado, conforme descrito no Quadro 1. Todos os refrigerantes avaliados foram adquiridos nos supermercados da cidade de João Pessoa e armazenados conforme instruções do fabricante.

Coleta de Dados Químicos dos Refrigerantes

As medidas de $\mathrm{pH}$ e titulação ácida (TA) foram realizadas imediatamente após a abertura de cada bebida à temperatura ambiente $\left(37^{\circ} \mathrm{C}\right)$.

$\mathrm{O} \mathrm{pH}$ inicial foi mensurado utilizando um medidor de $\mathrm{pH}$ (Orion $290^{\mathrm{a}}+$ - Thermo Electron Corporation), previamente calibrado, acoplado a um eletrodo imerso em $50 \mathrm{ml}$ da bebida dispensada em um bequer sob agitação, sem aquecimento, até a estabilização da sua leitura.

A titulação ácida (TA) foi realizada pela adição de incrementos de $0,2 \mathrm{ml}$ de solução padrão básica de $1 \mathrm{M} \mathrm{NaOH}$ sob agitação, até ser atingido os pHs 5,5 e 7,0. O volume total da solução padrão foi registrado. As medições foram feitas em triplicata e a média obtida foi utilizada. A capacidade tampão ( $\beta$ ) foi calculada de acordo a formula proposta por Lussi et al. ${ }^{11}$ :, onde $\Delta C$ é a quantidade total de base usada para elevar o $\mathrm{pH}$ inicial para 7,0 e $\circ \Delta \mathrm{pH}$ é a mudança no pH da solução.

\section{Análise Estatística}

Os resultados foram avaliados, utilizando-se estatísticas descritiva e inferencial. Os dados apresentaram uma distribuição normal (Shapiro Wilker), permitindo a utilização de testes paramétricos. Foram realizados os testes: ANOVA, seguido pelo teste de Bonferroni e teste t. As correlações entre as variáveis foram feitas com o teste de correlação de Pearson. O nível de significância considerado foi de $95 \%(p<0,05)$. O programa SPSS versão 19 foi utilizado para as análises.

\section{RESULTADOS}

As propriedades químicas investigadas das bebidas analisadas estão apre- 
sentadas na tabela 1 e gráfico 1 . Todos os refrigerantes estudados foram ácidos com o pH variando de 2,37 (sabor água tônica) a 3,35 (sabor laranja) para os convencionais, e de 2,52 (sabor água tônica) a 3,11 (sabor cola) para os orgânicos. Aquantidade de base $(1 \mathrm{~mol} / \mathrm{L} \mathrm{NaOH})$ necessária para neutralizar os refrigerantes, após a abertura, foi maior para os orgânicos do que para os convencionais, exceto para o sabor água tônica. Com relação à capacidade tampão, o padrão foi similar à titulação ácida, tendo o maior valor 15,61 para o refrigerante de limão orgânico (12-LIO) e o menor, de 5,29 para o guaraná convencional (5-GC).

Quando os refrigerantes orgânicos e convencionais foram analisados por sabor (cola, guaraná, laranja, limão e/ou água tônica), verificou-se diferenças estatisticamente significantes entre os mesmos, para a maioria dos grupos considerados. Na variável $\mathrm{pH}$, por exemplo, exceto o sabor guaraná, os demais apresentaram diferenças estatisticamente significativas entre a versão orgânica e a convencional. Considerando a TA para o pH 7.0, apenas os sabores cola zero e água tônica não apresentaram diferenças estatisticamente significativas entre as versões avaliadas. Além destes sabores, o guaraná zero também não apresentou diferença no que concerne a CT.

No gráfico 2, os sabores foram agrupados em dois grupos distintos: orgânicos e convencionais, sendo possível constatar que não houve diferenças estatisticamente significantes entre os mesmos, para todas as variáveis analisadas.

Correlações significativas foram observadas entre o $\mathrm{pH}$ e $\mathrm{NaOH} 7.0(r=-0,492, \mathrm{p}=$ $0,01)$; pH e CT ( $r=-0,384, p=0,012) ; \mathrm{NaOH}$ 7.0 e CT $(r=0,991, p=0,000)$.

\section{DISCUSSÃO}

Evidências crescentes têm demonstrado que o consumo excessivo de bebidas e alimentos ácidos contribui para o desenvolvimento da erosão dentária $a^{6,8,11,19}$. Diversas pesquisas apresentam dados comparativos sobre o potencial erosivo de diversas bebidas ácidas ${ }^{6,12,13,20}$. E, neste sentido, o presente trabalho possibilitou estimar o potencial erosivo de refrigerantes orgânicos, não descritos na literatura, pela determinação do $\mathrm{pH}$, titulação ácida e capacidade tampão.

Os refrigerantes são bebidas consumidas em larga escala em todo o mundo, mas as mudanças de hábitos e a procura por alimentos mais saudáveis e naturais têm alterado o padrão de consumo do mercado atual ${ }^{16}$. O consumo de produtos orgânicos tem sido motivado pela preocupação com o bem-estar com apelo emocional junto aos consumidores, associado ao fato de não terem adicionais químicos na sua produção e serem considerados seguros e saudáveis ${ }^{21}$.

Por se tratar de uma bebida inovadora que vem despertando o interesse do consumidor, a análise do $\mathrm{pH}$ dos refrigerantes orgânicos se torna relevante, sendo esta uma das características influenciadoras do potencial erosivo de algumas bebidas. Neste aspecto, considerando que valores de $\mathrm{pH}$ abaixo do considerado crítico para a dissolução do esmalte (5.5) deixam o mesmo vulnerável à dissolução ${ }^{7}$, os achados do presente estudo alertam para o potencial erosivo dos refrigerantes orgânicos. Os valores de $\mathrm{pH}$ encontrados para os refrigerantes convencionais neste estudo, corroboram os achados de Zimmer et al. ${ }^{2}$, Wang et al. ${ }^{7}$, Lussi et al. ${ }^{11}$ e Korte et al. ${ }^{22}$. 
Quadro 1. Informações básicas dos refrigerantes analisados nesse estudo de acordo com sabor, composição e fabricante

\begin{tabular}{|c|c|c|}
\hline Grupo & Sabor (tipo) & Ingredientes \\
\hline $1-C C$ & $\begin{array}{l}\text { Cola Conven- } \\
\text { cional }\end{array}$ & $\begin{array}{l}\text { Água gaseificada, açúcar, extrato de noz de cola, cafeína, corante caramelo IV, } \\
\text { acidulante ácido fosfórico e aroma natural. }\end{array}$ \\
\hline 2-CO & $\begin{array}{l}\text { Cola Orgâ- } \\
\text { nico }\end{array}$ & $\begin{array}{l}\text { Água gaseificada, açúcar demerara orgânico, extratos naturais de malte e noz de } \\
\text { cola, extrato de guaraná orgânico, acidulante natural ácido cítrico, aroma natural } \\
\text { e cafeína. Alérgicos: contém derivados de cevada. }\end{array}$ \\
\hline $3-C Z C$ & $\begin{array}{c}\text { Cola Zero } \\
\text { Convencional }\end{array}$ & $\begin{array}{l}\text { Água gaseificada, extrato de noz de cola, cafeína, aroma natural, corante caramelo } \\
\text { IV, acidulante ácido fosfórico, edulcorantes ciclamato de sódio }(27 \mathrm{mg}) \text {, acesulfame } \\
\text { de potássio }(15 \mathrm{mg}) \text { e aspartame }(12 \mathrm{mg}) \text { por } 100 \mathrm{ml} \text {, conservador benzoato de } \\
\text { sódio, regulador de acidez citrato de sódio. }{ }^{*} \text { Contém Fenilalanina }\end{array}$ \\
\hline 4-CZO & $\begin{array}{l}\text { Cola Zero } \\
\text { Orgânico }\end{array}$ & $\begin{array}{l}\text { Água gaseificada, edulcorantes naturais maltitol }(4 \mathrm{~g} / 100 \mathrm{ml}) \text {, eritritol }(2 \mathrm{~g} / 100 \mathrm{ml}) \text { e } \\
\text { glicosídeos de esteviol ( } 40 \mathrm{mg} \text { por } 100 \mathrm{ml}) \text {, concentrado de malte, extrato natural de } \\
\text { noz de cola, extrato de guaraná orgânico, acidulante natural ácido cítrico, aromas } \\
\text { naturais e cafeína. Alérgicos: contém derivados de cevada. }\end{array}$ \\
\hline $5-G C$ & $\begin{array}{c}\text { Guaraná } \\
\text { Convencional }\end{array}$ & $\begin{array}{l}\text { Água gaseificada, açúcar, extrato de guaraná, acidulante ácido cítrico, } \\
\text { conservadores: benzoato de sódio e sorbato de potássio, aromatizante e 6corante } \\
\text { caramelo IV. Não contém glúten. }\end{array}$ \\
\hline 6-GO & $\begin{array}{l}G \\
\mathrm{O}\end{array}$ & $\begin{array}{l}\text { Água gaseificada, açúcar demerara orgânico, extrato de guaraná orgânico, } \\
\text { acidulante natural ácido cítrico, aroma natural e corante natural de caramelo. }\end{array}$ \\
\hline 7-GZC & $\begin{array}{l}\text { Guaraná Zero } \\
\text { Convencional }\end{array}$ & $\begin{array}{l}\text { Água gaseificada, extrato de guaraná, acidulante ácido cítrico, edulcorantes, } \\
\text { aspartame }(34,8 \mathrm{mg} / 100 \mathrm{ml}) \text { e acesulfame de potássio }(9,0 \mathrm{mg} / 100 \mathrm{ml}) \text {, Conserva- } \\
\text { dor benzoato de sódio, aromatizante e corante caramelo IV. Não contém glúten } \\
\text { - contém fenilalanina. }\end{array}$ \\
\hline 8-GZO & $\begin{array}{l}\text { Guaraná Zero } \\
\text { Orgânico }\end{array}$ & $\begin{array}{l}\text { Água gaseificada, edulcorantes naturais eritritol e glicosídeos de esteviol }(40 \mathrm{mg} \\
\text { por } 100 \mathrm{ml}) \text {, extrato de guaraná orgânico, acidulante natural ácido cítrico, aroma } \\
\text { natural e corante natural de caramelo. }\end{array}$ \\
\hline 9-LAC & $\begin{array}{l}\text { Laranja Con- } \\
\text { vencional }\end{array}$ & $\begin{array}{l}\text { Água gaseificada, açúcar, sucos concentrados de laranja e maçã, vitamina C, } \\
\text { reguladores de acidez ácido cítrico e citrato de sódio, aroma sintético idêntico } \\
\text { ao natural, sequestrantes hexametafosfato de sódio e edta cálcio dissódico, } \\
\text { conservadores benzoato de sódio e sorbato de potássio, edulcorantes sucralose } \\
(4,2 \mathrm{mg}) \text { e acesulfame de potássio }(3,2 \mathrm{mg}) \text { por } 100 \mathrm{ml} \text {, corante artificial amarelo } \\
\text { crepúsculo FCF. }\end{array}$ \\
\hline 10-LAO & \begin{tabular}{|l} 
Laranja Orgâ- \\
nico
\end{tabular} & $\begin{array}{l}\text { Água gaseificada, açúcar orgânico, sucos orgânicos concentrados de tangerina } \\
\text { e laranja, acidulante natural ácido cítrico, aromas naturais e palm caroteno. }\end{array}$ \\
\hline 11-LIC & $\begin{array}{l}\text { Limão Con- } \\
\text { vencional }\end{array}$ & $\begin{array}{l}\text { Água gaseificada, suco de limão, acidulante ácido cítrico, aroma natural, } \\
\text { edulcorantes ciclamato de sódio }(72 \mathrm{mg}) \text { e sacarina de sódio }(11 \mathrm{mg}) \text { por } 100 \\
\text { ml, conservadores benzoato de sódio e sorbato de potássio, estabilizante citrato } \\
\text { de sódio. }\end{array}$ \\
\hline 12-LIO & $\begin{array}{l}\text { Limão Orgâ- } \\
\text { nico }\end{array}$ & $\begin{array}{l}\text { Água gaseificada, açúcar orgânico, suco de limão orgânico, acidulante natural } \\
\text { ácído cítrico, aroma natural e corante natural de clorofila. }\end{array}$ \\
\hline 13-ATC & $\begin{array}{l}\text { Água Tônica } \\
\text { Convencional }\end{array}$ & $\begin{array}{l}\text { Água gaseificada, açúcar, quinino, acidulante ácido cítrico, aroma natural e } \\
\text { conservador benzoato de sódio. }\end{array}$ \\
\hline $14-A^{-}$ & $\begin{array}{l}\text { Água Tônica } \\
\text { Orgânica }\end{array}$ & $\begin{array}{l}\text { gua gaseificada, açúca } \\
\text { sidulante natural ácido }\end{array}$ \\
\hline
\end{tabular}

(Fonte: Dados próprios, 2019) 
Tabela 1. Médias e desvio padrão dos valores de pH, titulação ácida para o pH 7.0 e Capacidade tampão dos grupos de refrigerantes analisados quanto a forma de produção convencional e orgânica, e mesmo sabores

\begin{tabular}{|c|c|c|c|c|}
\hline Variáveis & SABOR & $\begin{array}{c}\text { Convencionais } \\
\text { Média (DP) }\end{array}$ & $\begin{array}{l}\text { Orgânicas } \\
\text { Média (DP) }\end{array}$ & Valor de $\mathrm{p}^{*}$ \\
\hline \multirow{7}{*}{$\mathrm{pH}$} & Cola & $2,44( \pm 0,05)$ & $3,11( \pm 0,01)$ & 0,000 \\
\hline & Cola zero & $2,99( \pm 0,01)$ & $2,62( \pm 0,01)$ & 0,000 \\
\hline & Guaraná & $2,84( \pm 0,02)$ & $2,78( \pm 0,02)$ & 0,316 \\
\hline & Guaraná zero & $2,97( \pm 0,02)$ & $2,60( \pm 0,02)$ & 0,000 \\
\hline & Laranja & $3,35( \pm 0,03)$ & $2,75( \pm 0,01)$ & 0,000 \\
\hline & Limão & $2,88( \pm 0,05)$ & $2,65( \pm 0,02)$ & 0,000 \\
\hline & Água Tônica & $2,37( \pm 0,05)$ & $2,52( \pm 0,01)$ & 0,000 \\
\hline \multirow{7}{*}{ TA 7.0} & Cola & $1,26( \pm 0,17)$ & $1,68( \pm 0,08)$ & 0,044 \\
\hline & Cola zero & $1,11( \pm 0,18)$ & $1,47( \pm 0,03)$ & 0,139 \\
\hline & Guaraná & $1,14( \pm 0,10)$ & $1,78( \pm 0,05)$ & 0,000 \\
\hline & Guaraná zero & $1,09( \pm 0,05)$ & $1,55( \pm 0,05)$ & 0,015 \\
\hline & Laranja & $1,51( \pm 0,08)$ & $2,29( \pm 0,03)$ & 0,000 \\
\hline & Limão & $2,45( \pm 0,19)$ & $3,64( \pm 0,09)$ & 0,000 \\
\hline & Água Tônica & $3,88( \pm 0,17)$ & $3,61( \pm 0,31)$ & 0,523 \\
\hline \multirow{7}{*}{ CT } & Cola & $5,40( \pm 0,74)$ & $8,33( \pm 0,43)$ & 0,000 \\
\hline & Cola zero & $5,43( \pm 0,84)$ & $6,51( \pm 0,11)$ & 0,598 \\
\hline & Guaraná & $5,36( \pm 0,47)$ & $8,12( \pm 0,24)$ & 0,000 \\
\hline & Guaraná zero & $5,29( \pm 0,28)$ & $6,82( \pm 0,20)$ & 0,131 \\
\hline & Laranja & $8,06( \pm 0,39)$ & $10,32( \pm 0,10)$ & 0,004 \\
\hline & Limão & $11,34( \pm 0,91)$ & $15,61( \pm 0,44)$ & 0,000 \\
\hline & Água Tônica & $15,53( \pm 0,45)$ & $15,05( \pm 1,25)$ & 0,999 \\
\hline
\end{tabular}

*diferença significante entre os grupos convencionais e orgânicos $(p<0.05)$

Ao comparar os refrigerantes orgânicos com os convencionais, foi verificado que os sabores orgânicos tipo Cola e água tônica apresentaram valores de $\mathrm{pH}$ inferiores aos convencionais, contudo apenas o sabor guaraná demonstrou diferença estatisticamente significativa $(p<0,05)$. No entanto, quando da análise de todos os refrigerantes subdivididos em dois grupos, orgânicos e convencionais, a diferença entre as médias de $\mathrm{pH}$ não foi significativa. É importante ressaltar ainda que o pH, por si só, não é uma medida suficiente para se determinar o potencial erosivo de uma bebida ${ }^{2}$, pois precisa estar associada a titulação ácida e capacidade tampão .

No que diz respeito à titulação ácida (TA) dos refrigerantes estudados, foi verificado que os orgânicos necessitaram de uma maior quantidade de base para serem neutralizados. Estes achados apresentaram-se estatisticamente significativos quando comparadas as versões, orgânica e convencional de mesmo sabor, com exceção do sabor água tônica. É importante enfatizar que, conforme outros estudos $^{9,23}$ já desenvolvidos, a titulação dos refrigerantes convencionais se mostrou mais 
Gráfico 1. Médias e desvio padrão dos valores de pH, Titulação Ácida (TA) e Capacidade Tampão (CT) de todas as bebidas analisadas

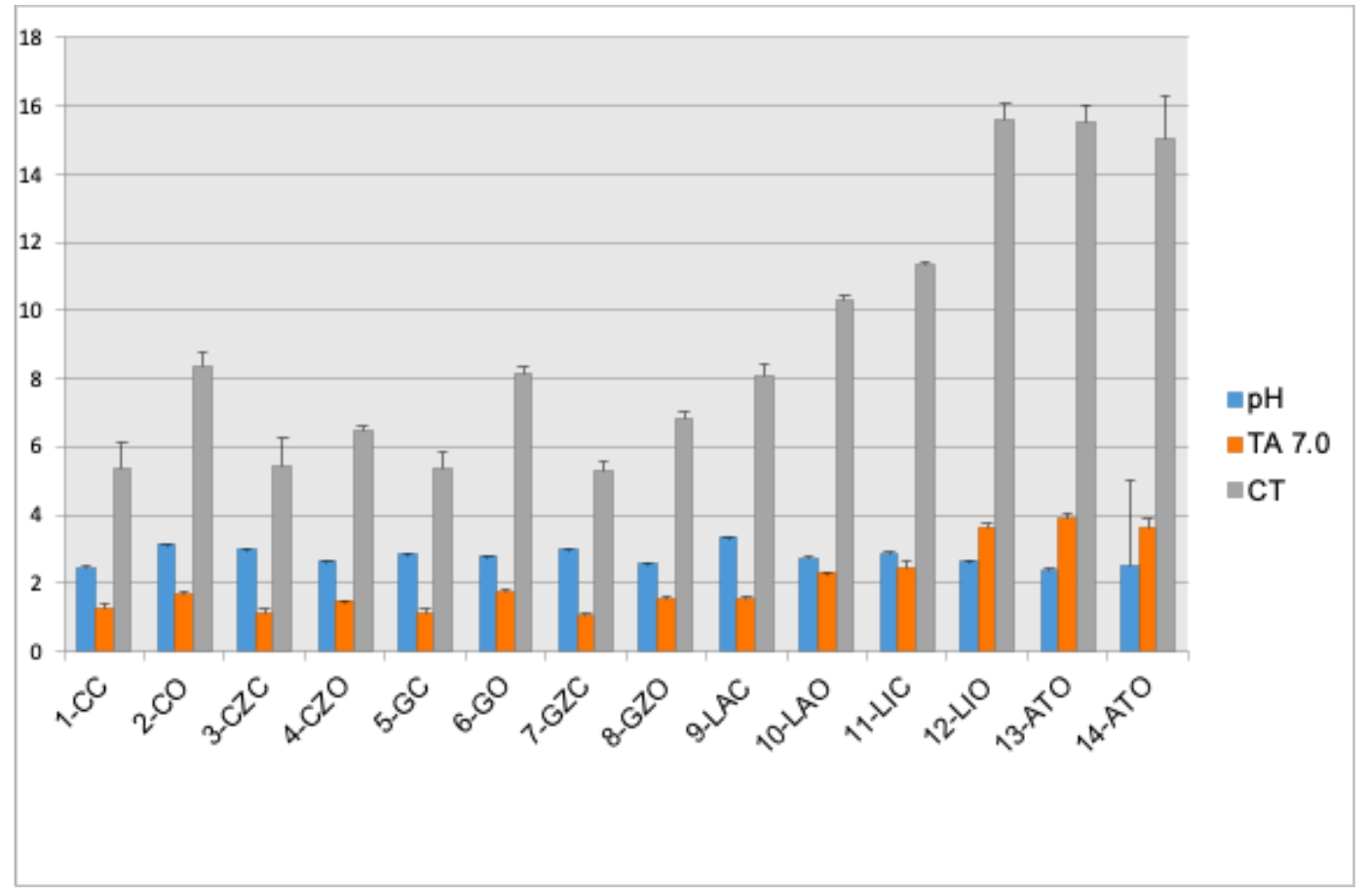

Gráfico 2. Distribuição das médias dos valores de pH, TA e CT dos refrigerantes orgânicos e convencionais

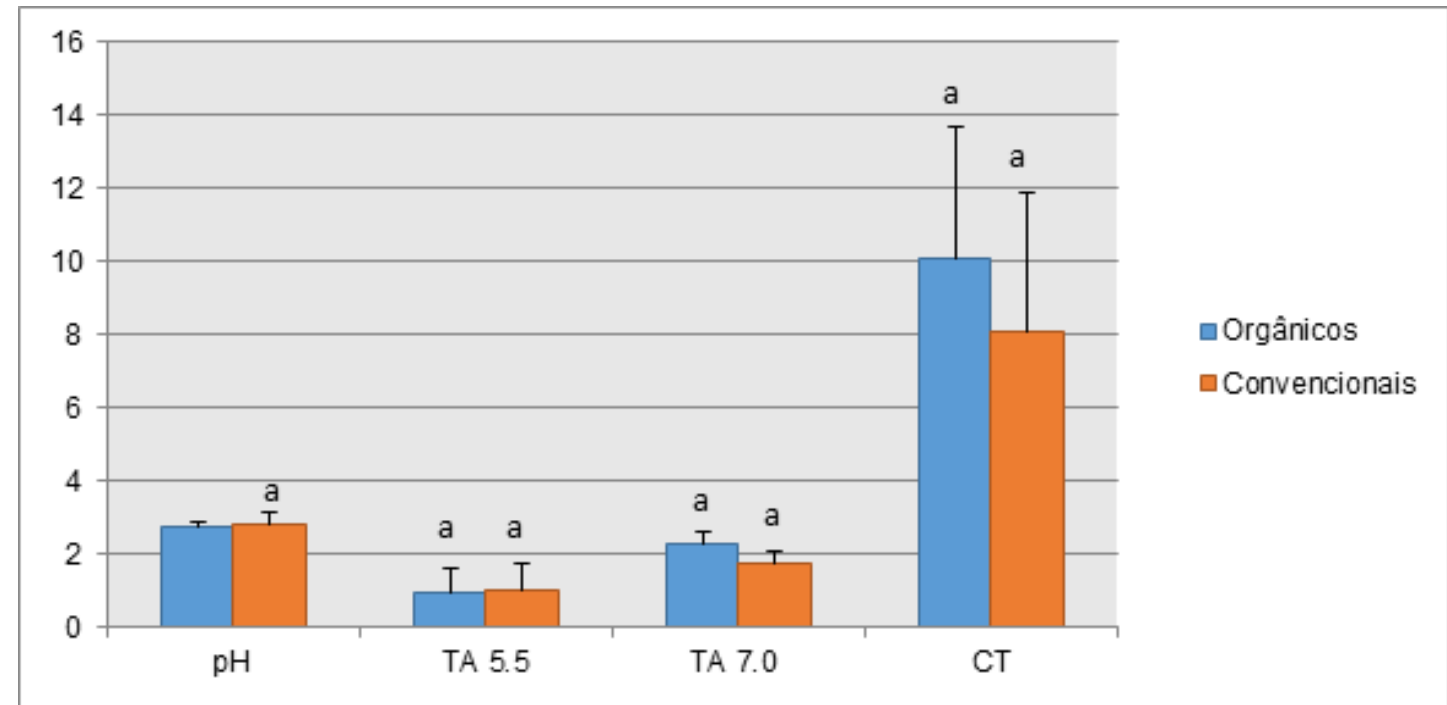

*Médias seguidas de mesma letra não diferem estatisticamente entre si (ANOVA, $p<0,05)$ para a mesma variável. 
alta do que outras bebidas ácidas. Esses achados precisam ser considerados visto que quanto maior a acidez titulável de uma solução, maior o seu potencial erosivo ${ }^{9,12}$. Além disso, a TA é uma medida, que pode ser utilizada para estimar o potencial erosivo de refrigerantes, quando o tempo de desafio ácido for aumentado 5 .-

Em estudos prévios, o sabor cola sempre apresentou um valor considerado alto de capacidade tampão (CT), mostrando ser uma bebida difícil de ser tamponada pela saliva e exibindo um potencial erosivo conhecido para a hidroxiapatita do esmalte ${ }^{8,24}$. No entanto, na presente pesquisa, o tipo cola convencional apresentou valores de CT abaixo de todos os orgânicos, bem como dos sabores laranja, limão e água tônica convencionais. Uma hipótese para este achado é que o acidulante natural de ácido cítrico seja mais resistente que o sintético, presente nos refrigerantes convencionais. O estudo de KORT et al ${ }^{22}$ também relata que os refrigerantes que contêm adoçantes de baixa caloria são mais erosivos do que os açucarados.

As propriedades químicas analisadas no presente estudo constituem importantes parâmetros para explicar o potencial erosivo dos refrigerantes orgânicos, contudo não se deve excluir a importância de associar esses dados ao tempo de exposição ao qual são submetidas enquanto desafio ácido, face às possíveis alterações que possam ocorrer na superfície do esmalte dentário ${ }^{14}$.

Diante dos achados encontrados, verifica-se a necessidade da realização de novos estudos com diversificação dos tempos de exposição, além da presença ou não de saliva, utilizando modelos de ciclagem variados.

Os achados deste estudo são relevantes à medida que visam despertar o interesse da comunidade científica para o desenvolvimento de pesquisas voltadas para a análise mais detalhada do potencial erosivo dos refrigerantes orgânicos, os quais estão sendo apresentados à população, como vantajosos para a saúde. Os resultados sugerem evidências de que a relação observada entre uma melhora subjetiva do bem-estar e o consumo de produtos orgânicos, neste caso, seja consequência de um efeito de rótulo, ou apenas, de efeito comercial.

\section{CONCLUSÃO}

A partir dos achados da presente pesquisa, pode-se concluir que os tanto os refrigerantes orgânicos como os convencionais são bebidas ácidas Pela análise das variáveis químicas, os refrigerantes orgânicos foram potencialmente mais erosivos que os convencionais, quando analisados individualmente de acordo com o sabor estudado. Dentre os sabores testados, o de limão orgânico apresentou-se como o mais erosivo, pelo seu baixo $\mathrm{pH}$, sua maior TA e CT, evidenciando que a saliva terá maior dificuldade de tamponá-lo no meio bucal.

Assim, os resultados encontrados foram alarmantes e devem ser estudados de maneira mais detalhada, visto que a procura por alimentos mais saudáveis e naturais tem aumentado nos últimos anos. É importante enfatizar que o consumo de produtos orgâ- 
nicos deve ser estimulado, no entanto para a variável erosão dentária, a determinação e investigação do potencial erosivo é de suma importância para que as orientações à população, quanto ao consumo e frequencia sejam adequadas.

\section{REFERÊNCIAS}

1. Oliveira AFB, Mathews SM, Ramalingam K, Amaechi B. The effectiveness of an NaF rinse containing fTCP on eroded enamel remineralization. J Public Heal. 2016; 24(2):147-152.

2. Zimmer S, Kirchner G, Bizhang M, Benedix M. Influence of Various Acidic Beverages on Tooth Erosion. Evaluation by a New Method. PLoS ONE, 2015; 10(6):1-8.

3. Luciano LCO, Ferreira MC, Paschoal MA. Prevalence and factors associated with dental erosion in individuals aged 12-30 years in a northeastern Brazilian city. Clin Cosmet Investig Dent. 2017; 16(9):85-91.

4. Salas MM, Nascimento GG, Huysmans MC, Demarco FF Estimated prevalence of erosive tooth wear in permanent teeth of children and adolescents: An epidemiological systematic review and metaregression analysis. J Dent. 2014; 43(1):42-50.

5. Comar LP, Salomão PMA, Souza BM, Magalhães AC Dental erosion: an overview on definition, prevalence, diagnosis and therapy. Braz Dent Sci 2013; 16(1):6-17.

6. Tocolini DG, Dalledone M, Brancher JA, de Souza JF, Gonzaga CC. Evaluation of the erosive capacity of children's beverages on primary teeth enamel: An in vitro study. J Clin Exp Dent. 2018;10(4):e383-7.

7. Wang $\mathrm{Y}$, Chang $\mathrm{C}$, Chi $\mathrm{C}$, Chang $\mathrm{H}$, Chiang $\mathrm{Y}$, Chuang $\mathrm{Y}$ et al. Erosive potential of soft drinks on human enamel: An in vitro study J. Formos. Med. Assoc. 2014; 113(11):850-856.

8. Oliveira AFB, Sampaio FC, Meira IA, Bezerra MGPG, Fernandes NLS, Paiva VMS. Erosive Potential of Industrializes Teas: An in vitro study. Pesq Bras Odontoped Clin Integr. 2017; 17(1):1-7.

9. Lussi A, Schlueter N, Rakhmatullina E, Ganss C. Dental erosion - an overview with emphasis on chemical and histopathological aspects. Caries Res. 2011; 45 (Suppl. 1): 2-12.

10. Jeong M, Jeong S, Son J, Chung S, Kim A, Kang E et al. A Study on the Enamel Erosion Caused by Energy Drinks. J Dent Hyg Sci. 2014; 14(4):597-609.

11.
12. Lussi A, Megert B, Shellis RP, Wang X. Analysis of the erosive effect of different dietary substances and medications. Br J Nutr. 2012; 107:252-262.

13. Barbour ME, Lussi A, Shellis RP. Screening and prediction of erosive potential. Caries Res. 2011; 45(suppl 1):24-32.

14. Hara AT, Zero DT. Analysis of the erosive potential of calcium-containing acidic beverages. Am. J. Dent. 2006, 19(6):319-325.

15. OstrowskaA, Szymański W, KołodziejczykŁ, Bołtacz-Rzepkowska E. Evaluation of the Erosive Potential of Selected Isotonic Drinks: In Vitro Studies. Adv Clin Exp Med. 2016; 25(6):1313-1319.

16. Reddy A, Norris DF, Momeni SS, Waldo B, DM D, Ruby JD. The $\mathrm{pH}$ of beverages in the United States. J. Am. Dent. Assoc. 2016. 147(4):255-263.

17. Oliveira FCR, Hoffmann R. Consumo de alimentos orgânicos e light/diet no Brasil. Segur. Aliment. Nutr. 2015; 22(1):541-557.

18. Bouguini RG, Torres EAFS. Alimentos orgânicos: qualidade nutritiva e segurança do alimento. $\quad \mathrm{S}$ e g u $\mathrm{r}$. Aliment. Nutr. 2006; 13(2):64-75.

19. Ministério da Agricultura, Pecuária e Abastecimento (BR). Orgânicos. O que são produtos orgânicos? [internet]. 2019.

20. Santos EJLD, Meira IA, Sousa ETD, Amaechi BT, Sampaio FC, Oliveira AFB. Erosive potential of soy-based beverages on dental enamel. Acta Odontologica Scandinavica, 2019; 77(5):1-7.

21. Jager DHJ, Vieira AM, Ruben JL, Huysmans MCDNJM. Estimated erosive potential depends on exposure time. J. Dent. 2012; 40(12):1103-1108.

22. Medeiros BE, Marconato T, Souza AE. Estudo bibliométrico sobre a motivação para o consumo de produtos orgânicos. Cad. Econ. 2017; 21(38):74-88.

23. Korte A, Angelopoulou MV, Maroulakos G. Assessing the Effect of Low Calorie Soda Beverages on Primary Tooth 
Enamel: An In Vitro Study, J Clin Pediatr Dent. 2019; 43(3): 190-195.

24. Murrella S, Marshall TA, Moynihan PJ, Qian F, Wefel JS. Comparison of in vitro erosion potentials between beverages available in the United Kingdom and the United States. J. Dent. 2010; 38(4):284-289.

25. Barac R, Gasic J, Trutic N, Sunaric S, Popovic J, Djekic $P$ et al. Erosive Effect of Different Soft Drinks on Enamel Surface in vitro: Application of Stylus Profilometry. Medical Principles and Practice. 2015; 24(5):451-457.

\section{Correspondência}

João Paulo Gomes Martins

Rua Farmacêutico Antônio Leopoldo Batista, 193,

Ed. Apiaí, apto 304, Jardim São Paulo, CEP: 58.051-110 João Pessoa, Paraíba, Brasil.

Email: joaopaulonomundo@gmail.com 